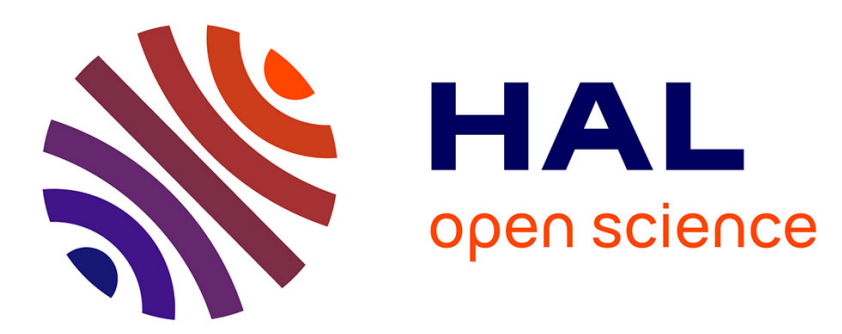

\title{
Gouverner l'Église catholique. Pouvoir hiérarchique et construction doctrinale
}

\author{
Jacques Palard
}

\section{To cite this version:}

Jacques Palard. Gouverner l'Église catholique. Pouvoir hiérarchique et construction doctrinale. Archives de Sciences Sociales des Religions, 2018, 184, pp.119-133. 10.4000/assr.38959 . halshs02469412

\section{HAL Id: halshs-02469412 \\ https://shs.hal.science/halshs-02469412}

Submitted on 26 Jan 2022

HAL is a multi-disciplinary open access archive for the deposit and dissemination of scientific research documents, whether they are published or not. The documents may come from teaching and research institutions in France or abroad, or from public or private research centers.
L'archive ouverte pluridisciplinaire HAL, est destinée au dépôt et à la diffusion de documents scientifiques de niveau recherche, publiés ou non, émanant des établissements d'enseignement et de recherche français ou étrangers, des laboratoires publics ou privés. 


\section{Archives de sciences sociales des religions}

184 | octobre-décembre 2018

Bulletin bibliographique

\section{Gouverner l'Église catholique. Pouvoir hiérarchique et construction doctrinale}

Governing the Catholic Church. Hierarchical power and doctrinal construction

Gobierno de la Iglesia Católica. Poder jerárquico y construcción doctrinal

Jacques Palard

\section{OpenEdition}

Journals

Édition électronique

URL : https://journals.openedition.org/assr/38959

DOI : $10.4000 /$ assr.38959

ISSN : $1777-5825$

Éditeur

Éditions de l'EHESS

Édition imprimée

Date de publication : 1 décembre 2018

Pagination : 119-133

ISSN : 0335-5985

Référence électronique

Jacques Palard, "Gouverner l'Église catholique. Pouvoir hiérarchique et construction doctrinale », Archives de sciences sociales des religions [En ligne], 184 I octobre-décembre 2018, mis en ligne le 01 janvier 2022, consulté le 12 janvier 2022. URL : http://journals.openedition.org/assr/38959 ; DOI https://doi.org/10.4000/assr.38959 
Jacques Palard

\title{
Gouverner l'Église catholique
}

\section{Pouvoir hiérarchique et construction doctrinale}

\author{
À propos de: \\ Chopelin Paul (éd.), Gouverner une Église en révolution. Histoires \\ et mémoires de l'épiscopat constitutionnel, Lyon, Laboratoire de \\ Recherche Historique Rhône-Alpes, coll. "Chrétiens et Sociétés. \\ Documents et Mémoires », 2017, 315 p. \\ Douyère David, Communiquer la doctrine catholique. Textes et conver- \\ sations durant le concile Vatican II d'après le journal d'Yves Congar, \\ Genève, Labor et Fides, coll. "Enquêtes ", 2018, 251 p. \\ Jankowiak François, Pettinaroli Laura (éds.), Les cardinaux entre \\ Cour et Curie. Une élite romaine, 1775-2015, Rome, École française \\ de Rome, 2017, 465 p. \\ SORREl Christian (éd.), Renouveau conciliaire et crise doctrinale. Rome \\ et les Églises nationales (1966-1968), Lyon, Laboratoire de Recherche \\ Historique Rhône-Alpes, coll. "Chrétiens et Sociétés. Documents \\ et Mémoires ", 2017, 406 p. \\ VARIN Roland, Le sacrement du ministère apostolique. La sacramentalité \\ de l'épiscopat et ses conséquences ecclésiologiques, Paris, Artège \\ Lethielleux, 2016, 317 p.
}

La période actuelle se prête sans doute de façon plus propice que d'autres à l'examen des rapports de pouvoir au sein de l'Église catholique. Le pape François, élu en 2013 à la suite de la renonciation de Benoît XVI, a pris depuis lors des positions inédites qui suscitent un surcroît d'intérêt pour l'examen de ces rapports au sein même, mais aussi en dehors, de l'organisation religieuse. Sa Lettre au peuple de Dieu publiée le 20 août 2018, dans le contexte de nouveaux dévoilements massifs de scandales pédophiles attribués à des membres du clergé, fait délibérément appel à une «réaction globale et communautaire ", c'est-à-dire à une "participation active de toutes les composantes du peuple de Dieu ». Associant abus sexuels, abus de pouvoir et abus de conscience, le Souverain pontife affirme que «dire non aux 
abus, c'est dire non, de façon catégorique, à toute forme de cléricalisme ». Le pape s'en prend à la pratique cléricale, identifiable à l'excès de pouvoir des membres du clergé, parce qu'elle «engendre une scission dans le corps ecclésial qui encourage et aide à perpétuer beaucoup des maux que nous dénonçons aujourd'hui ". Bien que conçue à d'autres fins et dans un esprit polémique évidemment différent, on croirait entendre comme en écho la célèbre apostrophe lancée par Léon Gambetta à la Chambre des députés le 4 mai 1877: «Le cléricalisme ? Voilà l'ennemi! » Dans la foulée de la lettre pontificale, le quotidien La Croix, dès le 30 août suivant, a consacré ses cinq premières pages à l'examen des modalités de lutte contre le cléricalisme par l'ouverture de dix "chantiers ", parmi lesquels: mettre à leur juste place les prêtres et les laïcs, rappeler l'égalité de tous devant le baptême, organiser des lieux de débat dans l'Église, gouverner les diocèses de manière plus collégiale, associer plus de femmes à la formation des prêtres et les placer à des fonctions d'autorité.

Cette actualité, qui donne à voir une véritable inversion du cours qu'adopte habituellement le mouvement de protestation ou de revendication au sein de l'Église catholique, permet de lire avec une curiosité renouvelée les ouvrages d'histoire moderne et contemporaine consacrés au fonctionnement ecclésiastique. C'est en effet aussi de l'ultime sommet de la hiérarchie que provient aujourd'hui, à l'encontre des "élites » cléricales, l'appel à la rénovation de l'institution et à un changement de culture. Afin d'échapper à tout anachronisme, cette actualité ne doit évidemment pas conduire à soustraire les ouvrages d'histoire ici retenus aux contextes et aux enjeux qu'ils prennent en compte, qu'il s'agisse de la gestion de l'universel ou de celle de configurations religieuses nationales ou locales... Cette saine prudence vaut singulièrement pour l'examen des périodes de crise ou de changement radical.

\section{Épiscopat et cardinalat}

C'est précisément à une crise profonde et inédite que se trouve confronté l'épiscopat constitutionnel sous la Révolution française, épiscopat dont les membres, selon les termes de Paul Chopelin, qui dirige l'ouvrage qui leur est consacré, "ont longtemps été victimes d'une légende noire (Chopelin, 2017: 7), d'une «damnatio memoriae» (ibid.: 14), dont traite Christian Sorrel à propos de l'évêque du Mont-Blanc, François-Thérèse Panisset, premier prélat constitutionnel à s'être rétracté publiquement (ibid.: 281-304). Si la figure de "l'abbé Grégoire ", évêque de Blois, a donné lieu à de nombreux travaux, tel n'a pas été le cas, jusqu'à une date récente, des prélats de la première Église constitutionnelle, née de la Constitution civile du clergé (CCC) adoptée le 12 juillet 1790 (et qui va conduire à la suppression de 52 évêchés), ni de ceux de la seconde, qui s'est organisée après Thermidor. Valérie GuitienneMurger note que, malgré le caractère douteux ou improbable du jansénisme de nombreux constitutionnels, le texte fondateur que constitue la CCC fut souvent compris comme l'œuvre même des jansénistes et donc âprement 
combattu par un clergé attaché à la restauration d'un «catholicisme de plus en plus romain» (ibid.: 262).

L'intérêt de l'ouvrage tient à la double attention portée par les auteurs à l'Église catholique en période révolutionnaire: leur regard porte non seulement sur des personnalités représentatives, mais aussi sur des situations institutionnelles et sur la pensée théologique, singulièrement en matière d'ecclésiologie. Les questions auxquelles répondent les contributions sont centrales: comment situer les évêques constitutionnels, élus lors de scrutins organisés par les administrations locales au début de l'année 1791, dans la longue histoire de l'épiscopat français? Quelles sont les conditions d'exercice de l'autorité épiscopale pendant la Révolution? Mais également, de quelles ressources disposent ces évêques pour faire front à la fois à l'hostilité de l'Église réfractaire, par définition accusatrice et concurrente, et celle des militants révolutionnaires, peu enclins à s'incliner devant des responsables religieux ou à leur apporter leur appui ? Isabelle Antunes observe que «l'évêque constitutionnel est impuissant face aux entreprises du clergé réfractaire» (ibid.: 53) et qu'il va rapidement perdre le soutien des autorités civiles; dès lors, «les jours de l'Église constitutionnelle en tant qu'Église d'État sont comptés» (ibid.: 55). Les difficultés que rencontre l'épiscopat constitutionnel tiennent à l'enjeu nodal que représente la conjonction entre engagement politique et responsabilité pastorale, statut de fonctionnaire public et ministère ecclésiastique. Caroline Chopelin-Blanc souligne que l'abbé Grégoire voit dans son double rôle d'évêque et de député - ce sera aussi le cas de 23 de ses collègues entre 1791 et 1795 - un vecteur favorable à la transposition de la "république d'inspiration ecclésiale à la sphère politique» (ibid.: 27). Dans la même ligne interprétative, Jean Dubray estime que selon l'auteur de l'Essai historique sur les libertés de l'Église gallicane (1818), qui fut le véritable chef de file de l'Église de France pendant une décennie, l'Église a pour finalité de présenter un modèle à la société civile et de "rappeler, constamment, l'inspiration religieuse des valeurs démocratiques" (ibid.: 127). C'est à la mise en œuvre de ce grand projet de démocratie intra-institutionnelle, qui s'est matérialisé par l'importance accordée à la collégialité épiscopale, que Joseph F. Byrnes porte son attention: entre 1795 et 1801, l'Église constitutionnelle «a organisé quatre-vingts synodes diocésains, huit conciles métropolitains et deux conciles nationaux» (ibid.: 181). En cela, aux yeux de Grégoire, la CCC aura initié, y compris sur le plan du gouvernement - lui aussi collégial - des diocèses de 1791 à 1793, un retour aux sources, celles d'une institution dans laquelle J. Dubray décèle la quête d'une "Église pré-constantinienne, et donc étrangère à toute espèce de concordat" (ibid.: 132; voir aussi Plongeron, 1973, 1989). Pareille option vaudra à Grégoire, à la différence de douze de ses confrères eux aussi constitutionnels, de ne pas être intégré dans le corps épiscopal au lendemain du Concordat. Parmi ces évêques figure Jean-François Périer qui avait été élu évêque de Clermont en février 1791 et presque aussitôt porté à la présidence du Conseil général du Puy-de-Dôme. Philippe Bourdin nous fait suivre son parcours mouvementé: après un exil grenoblois, provoqué par la 
radicalisation des dispositions anticatholiques des acteurs politiques locaux, il retrouve son siège épiscopal en novembre 1795. «Absout par le pape auquel il a envoyé une lettre d'allégeance " (Chopelin, 2017: 94), il quitte au printemps 1802 le Puy-de-Dôme pour le siège d'Avignon. On sait que les ressentiments associés au souvenir et aux effets du serment constitutionnel sont demeurés vivaces encore deux siècles plus tard: à l'occasion de la commémoration du bicentenaire de la Révolution française, et à la différence du nonce apostolique Mgr Antonetti, le cardinal Lustiger, archevêque de Paris, a refusé de se rendre, le 12 décembre 1989, à la cérémonie du transfert des cendres de l'abbé Grégoire au Panthéon.

Se risquer à des regards croisés entre sciences humaines ou sociales et sciences religieuses exige discernement et vigilance, pour des raisons qui ne relèvent pas du seul ordre épistémologique. L'analyse qui suit est donc placée sous le signe de la prudence et de la circonspection, d'autant que les investigations consacrées à la période révolutionnaire de l'histoire de l'Église catholique de France, non seulement troublée mais également marquée par de profonds antagonismes, tranchent on ne peut plus, en l'occurrence, avec le développement de pure théologie que Roland Varin (2016) consacre à «la sacramentalité de l'épiscopat». La perspective insiste ici sur la contribution quasi ontologique et essentialiste qu'apporte à la construction de l'Église le "sacrement du ministère apostolique », c'est-à-dire la consécration à une fonction institutionnelle dont le fondement prend son origine dans l'appel par Jésus des douze apôtres, d'où, précisément, le qualificatif " apostolique ». En parfait contrepoint de l'étude historique de l'épiscopat constitutionnel, cette perspective s'inscrit à proprement parler dans une approche de théologie dogmatique, que l'on pourrait dire hors-sol, dans la mesure où les conditions d'exercice de la fonction épiscopale ne sont jamais prises en considération, mais non pour autant a-historique. Toutefois, sur ce point même, et pour au moins deux épisodes majeurs, la vision linéaire du développement théologique et dogmatique de la sacramentalité de l'épiscopat mérite d'être interrogée. Écrire que «le concile Vatican II entend d'abord compléter l'enseignement de Vatican I qui n'a pu traiter que de la papauté » (ibid.: 21), c'est se faire oublieux des conditions historiques du vote du dogme de l'infaillibilité pontificale, en contexte de forte prégnance de l'ultramontanisme: une soixantaine d'évêques, dont Mgr Dupanloup, opposés à l'adoption de ce texte, ont en effet alors quitté Rome pour ne pas avoir à s'opposer formellement à l'adoption du texte. En outre, évoquer tout aussitôt que «Vatican II va lui aussi traiter de l'épiscopat et définir sa sacramentalité au sein d'une constitution sur l'Église, Lumen Gentium » (ibid.: 21), sans faire état du total changement de paradigme et d'argumentaire auquel a été soumis, à l'encontre de la Curie, la première version du texte, c'est, à l'évidence, ne s'attacher qu'à la version finale de cette production dogmatique alors qu'en l'affaire le processus est probablement au moins aussi instructif que le résultat. Après un chapitre consacré à la nature de l'Église, la première version de ce texte, remis aux Pères du Concile en novembre 1962 sous le titre De Ecclesia, consacrait en 
effet son chapitre 2 à l'organisation hiérarchique de l'Église; dans la version finale, cette partie du texte se trouve reportée en troisième position, après le nouveau chapitre 2, désormais consacré au «Peuple de Dieu». Dans le journal qu'il a tenu tout au long du Concile, le Père Congar rapporte ces propos prononcés sur un ton impératif le 30 septembre 1963 par Mgr Gargitter, évêque de Brixen (Italie de langue allemande) et qui sont significatifs de l'inversion à laquelle a été ainsi soumis le "schéma » de la Curie: "Qu'on rédige de façon plus organique, à la lumière de l'idée de peuple de Dieu; la hiérarchie elle-même doit sa première dignité au fait qu'elle lui appartient» (Congar, 2002: 409). Ce simple rappel historique de l'opinion de l'un des acteurs conciliaires, joint aux récentes injonctions du pape François dans sa lettre du 20 août 2018 et à leur déclinaison par le journal La Croix quelques jours plus tard, donne à penser que l'argumentaire de R. Varin, attaché à fonder dogmatiquement le pouvoir épiscopal, ne prend pas en compte les conflits et les négociations qui ont accompagné le processus conciliaire de production du dogme. Il s'intéresse moins encore aux enjeux organisationnels dont ce processus est porteur, et qui influent pourtant sur les modalités concrètes d'exercice du métier d'évêque. Or, selon Jacques Lagroye, le discours épiscopal «est directement affecté par les transformations rapides de la société ecclésiale et de la conception qu'ont les clercs du rapport entre l'Église et la société » (Lagroye, 1983 : 82). Sur le plan juridique, le Père Patrick Valdrini, alors doyen de la Faculté de droit canonique de l'Institut catholique de Paris, estime par ailleurs que «le droit canonique [doit] encore progresser vers la création d'institutions indiquant clairement que l'exercice du pouvoir ecclésiastique ne peut être arbitraire»(Valdrini, 1988: 127).

Entrer par le Collège des cardinaux, dénommé Sacré Collège jusqu'au Code de droit canonique de 1983, pour aborder l'étude de l'Église catholique permet d'engager une analyse comparative de nature spatio-temporelle du gouvernement ecclésiastique. C'est une telle perspective que développe l'ouvrage dirigé par François Jankowiak et Laura Pettinaroli (2017), puisque le terrain qu'il couvre est par définition planétaire et que la période étudiée part du dernier quart du XVIII ${ }^{\mathrm{e}}$ siècle pour ne trouver son terme que dans la période la plus récente. Il permet ainsi d'éclairer et de mieux comprendre une actualité religieuse marquée par la ferme volonté de l'actuel pontife romain de réformer une Curie qui s'est valu de sa part, quelques jours avant la fête de Noël 2014, des remontrances d'une rare violence. Le pape François a en effet alors diagnostiqué pas moins de "quinze maladies ", allant du «narcissisme " à la «schizophrénie existentielle» en passant par la « recherche insatiable du pouvoir». L'ouvrage aurait pu - dû ? - relater cet événement singulier, qui jette une lumière rétrospective sur les décennies passées et entend initier un mouvement de réforme; la vigueur des semonces explique en effet la commande faite par le pape au Conseil de neuf cardinaux - d'où son nom C9 - qu'il a créé un an plus tôt d'un projet de constitution apostolique visant à transformer la Curie. Les auteurs de ce projet, remis en juin 2018, se sont fondés sur trois "principes inspirateurs » : tradition, adaptation et coordination. 
L'ouvrage, qui présente 25 contributions, résulte d'un projet collectif consacré à l'étude de la fonction cardinalice et qui associe trois instances de recherche: l'École française de Rome, le centre Droit et Sociétés Religieuses de Paris-Saclay et l'unité de recherche Religion, Culture et Société de l'Institut catholique de Paris. Il fait suite à la publication, en 2015 et 2016, de premiers travaux dans les Mélanges de l'École française de Rome, Italie-Méditerranée (MÉFRIM). Ses coordonnateurs l'ont délibérément centré sur l'étude des cardinaux en tant qu'élite romaine, et plus précisément sur les relations que celle-ci entretient "avec le souverain pontife dans les cadres longtemps mêlés de la cour papale et de la Curie romaine» (ibid.: 5). Ils estiment que s'observe aujourd'hui encore une interpénétration entre Curie et cour pontificale.

Chacune des deux parties de l'ouvrage correspond à l'une des deux catégories de cardinaux: la première est celle des cardinaux de "siège » ou « résidentiels », que l'on peut également dire «non-romains» puisqu'ils sont à la tête d'un diocèse; ils représentent actuellement environ les deux tiers des quelque 120 cardinaux qui, parce qu'âgés de moins de 80 ans, peuvent siéger en conclave et voter pour l'élection d'un nouveau pape. Ils sont étudiés à la façon d'une "élite politique ( (ibid.: 6), à la charnière de Rome et des États. La seconde catégorie est constituée par les cardinaux dont la fonction s'exerce au sein de la Curie. Dans son étude des formes et des enjeux que revêt la "protection cardinalice" d'organisations d'action catholique féminine, Magali Della Sudda observe les conditions qui conduisent à choisir plutôt un «cardinal au plus près du Souverain Pontife» ou plutôt «l'ordinaire du siège de l'association" (ibid.: 137).

Le processus de sécularisation a conduit à un net reflux des négociations ou des interventions étatiques destinées à peser sur la désignation de nouveaux cardinaux; en France, la rupture des relations diplomatiques avec le SaintSiège en 1904 a joué de façon radicale en ce sens. Piero Doria, qui analyse l'évolution théologique du cardinalat sous Paul VI ainsi que la contribution des cardinaux au concile Vatican II, rappelle l'impossibilité de participer à cet événement dans laquelle s'est trouvé le cardinal hongrois Mindszenty, réfugié à l'ambassade américaine de Budapest de 1956 à 1971. Dans un tout autre registre, Marie Gayte souligne l'importante évolution de la place des cardinaux dans la vie sociale et politique des États-Unis. L'absence de relations diplomatiques entre Washington et le Saint-Siège de 1867 à 1984 a conduit à faire des cardinaux de véritables médiateurs diplomatico-religieux. Sans doute se sont-ils montrés critiques à l'encontre de la politique de défense de Reagan ou de son soutien à des forces de droite ou d'extrême droite au Nicaragua ou au Salvador, mais le phénomène le plus notable réside bien dans l'établissement des solides et durables relations qu'ils ont nouées avec les leaders du Parti républicain. Le cardinal Dolan de New York est ainsi venu prononcer la prière de bénédiction finale lors la convention républicaine qui s'est tenue à Tampa, en Floride, en août 2012 (ibid.: 107).

Le mouvement d'internationalisation du corps des cardinaux qui s'amorce avec le pontificat de Pie IX (1846-1878) connaît une nette amplification à 
partir du concile Vatican II. Audrey Virot note que lors du conclave qui a conduit à l'élection de Pie VI en 1775 on ne comptait que 4 participants non italiens sur 44, tandis que pour l'élection de Jean-Paul II en 1978 ils étaient 86 sur 111 (ibid.: 49). Selon Claude Prudhomme, l'ouverture internationale qui s'opère à compter du milieu des années 1960 s'inscrit «dans le contexte d'une décolonisation qui a fait surgir de nombreux États indépendants" (ibid.: 63). Mais l'auteur note également que le passage par une université romaine prestigieuse représente l'élément déterminant dans le choix des cardinaux entre 1946 et 1978 ; c'est dire que la socialisation romaine constitue un marqueur et un atout essentiel d'éligibilité.

L'analyse de la situation française, que présentent Frédéric Le Moigne et Christian Sorrel, apporte d'intéressantes informations sur l'influence qu'a exercée la période conciliaire comme cadre mais aussi facteur d'évolution de la fonction cardinalice. Cette contribution commence par la mention d'un coup de force, celui que tente et que réussit en octobre 1962 le cardinal Liénart, archevêque de Lille, en obtenant "le report des élections des commissions [conciliaires] verrouillées par la Curie romaine" (ibid.: 79). Les auteurs montrent également de quelle façon la collégialité épiscopale, qui conduit à la création de la Conférence des évêques de France, en 1964, opère une transformation du rapport de forces et des formes de légitimité, en l'occurrence "une dissociation entre la pourpre et la présidence de la conférence » (ibid.: 85), fonction qui devient en effet élective.

Les cardinaux de la Curie, auxquels est consacré le second volet de l'ouvrage, constituent, par leurs fonctions en matière de conseils et de décisions, l'élite administrative et le centre du pouvoir: les chefs de dicastères ont un accès direct au pape et sont en liens étroits avec l'appareil diplomatique du Saint-Siège. C'est évidemment, au premier chef, le cas pour le secrétaire d'État, qu'étudie Jean-Marc Ticchi au travers de la figure du cardinal Rampolla, étroit collaborateur de Léon XIII; cela est également vrai du pro-préfet de la Congrégation de la doctrine de la foi (l'ex-Saint-Office), poste qu'occupe le cardinal Ratzinger avant son élection au pontificat en 2005. On observe ici à nouveau que l'exercice de fonctions lors du concile a joué un rôle notable dans le processus de sélection cardinalice: François Weiser note que 43 experts conciliaires (parmi lesquels les théologiens français Congar, Daniélou et de Lubac) sont devenus cardinaux entre 1965 et 2014 (ibid.: 245); toutefois, un tiers d'entre eux occupait déjà un poste dans la Curie à la veille du concile. Cette filière a entraîné "une romanisation, ou plutôt une curialisation des structures post-conciliaires" (ibid.: 260). En contrepoint de ce processus de centralisation, Paul VI, dans son allocution à la Curie du 21 septembre 1963, annonce une réforme de cette instance, au nom de la nécessaire décentralisation du gouvernement de l'Église catholique. Ce projet prend partiellement effet au cours des années 1960 et 1970, qui voient certains dicastères confiés à des évêques résidentiels. Parmi les trois cas qui illustrent cette orientation, le plus notable est celui du cardinal Roy, archevêque de Québec, nommé par Paul VI à la tête tout à la fois de la Commission Justice et Paix, du Conseil 
pour les laïcs et du Comité pour la famille. Jean-Paul II ne reconduira pas pareille expérience - d'ailleurs demeurée limitée - de déconcentration plus que de décentralisation, dont Gilles Routhier observe qu'elle a entrainé un temps, du fait d'une activité multipolaire, "un nouveau rapport entre la Curie romaine et les Églises locales» (ibid.: 329).

Le portrait que l'ouvrage donne à voir et à suivre, c'est celui d'élites centrales d'un appareil gouvernemental qui se dote progressivement de ressources humaines aptes à une gestion internationale de ses «intérêts ", dans la ligne des trois préoccupations que J.-M. Ticchi retient du système d'action du secrétaire d'État Rampolla: «La volonté de protéger la dignité du Saint-Siège, le désir de tracer la limite entre le tolérable et l'intolérable dans les relations avec les gouvernements et le souhait d'encourager l'union dans l'Église» (ibid.: 371). C'est aussi ces préoccupations que met en exergue Olivier Sibre dans son examen de l'action diplomatique du Saint-Siège en contexte d'internationalisation. Si l'application du modèle gouvernemental à l'Église catholique, telle qu'y conduit l'étude de l'action des cardinaux, est analogiquement fondée, on ne saurait être surpris de relever la place qu'occupent les antagonismes entre les diverses administrations vaticanes; on en voit la manifestation, dans l'environnement de Pie XI, avec le conflit qui oppose le cardinal Merry del Val, patron du Saint-Office, et le cardinal Gasparri, secrétaire d'État: Marie Levant n'hésite pas à parler d'une "guerre à couteaux tirés» (ibid.: 312).

On peut regretter l'absence d'introductions à visée synthétique et transversale en ouverture de chacun des deux groupes de contributions. Les directeurs de l'ouvrage avancent le chiffre de 70 réponses à l'appel à communications, indicateur très sûr de l'intérêt que revêt le sujet et du dynamisme de la recherche en ce domaine. Le choix à des fins éditoriales n'a évidemment pu être que sélectif, voire drastique. Ce n'est sans doute pas la voie la meilleure pour construire une cohérence éditoriale, mais on retiendra surtout la grande diversité des objets, la richesse de la documentation et la pertinence des approches sur un terrain d'investigation dont il convient de rappeler l'actualité des enjeux. Dans sa conclusion, Philippe Levillain parle fort justement d'études «approfondies, à la fois érudites et savantes, c'est-à-dire pointues et conceptuelles » (ibid.: 396).

\section{La fabrique et la défense du dogme}

L'ouvrage de David Douyère (2018) aurait pu s'intituler «la fabrique du dogme », expression que l'auteur retient en conclusion pour rendre compte de sa démarche. Il vise en effet à analyser comment une organisation - en l'occurrence l'Église catholique - structure sa norme de pensée et d'action au travers de ses paroles et de ses écrits, ainsi qu'on a déjà pu en voir la traduction avec l'ouvrage de R. Varin. L'écriture à laquelle se livrent les acteurs ecclésiastiques de haut rang est en effet comprise comme "une instance normative qui vise à orienter la pensée sur le monde surnaturel et naturel qu'elle décrit. C'est là, précisément, sa dimension dogmatique» (ibid.: 113). Mutatis mutandis, cette démarche s'apparente à la réflexion qu'a conduite 
Ramsay MacMullen (2008), pour une période bien plus ancienne, dans son ouvrage au titre français évocateur: Voter pour définir Dieu.

Les paroles et les écrits auxquels s'attache David Douyère sont ceux du Concile Vatican II (1962-1965). Mais ces productions discursives - les échanges aussi bien que les textes - ne sont pas appréhendées dans leur version finale: l'auteur les saisit par le truchement de l'ouvrage posthume, évoqué plus haut, du Père Yves Congar (1904-1995), Mon Journal du Concile, fort de deux gros volumes d'environ 600 pages chacun et publié en 2002. D. Douyère dit clairement que son objectif n'est pas l'étude, en soi, du Concile; il entend bien plutôt centrer son analyse sur les processus communicationnels qui s'y sont développés et que le "journal » du Père Congar permet d'appréhender. $\mathrm{La}$ «communication» est entendue comme «la production et la mise en circulation de sens" (Douyère, 2018: 19).

Cette recherche, ainsi conduite sur « un terrain de papier» (ibid.: 39), mérite attention en raison à la fois de la haute stature intellectuelle du Père Congar, théologien dominicain novateur qui a subi quelques années auparavant, sous le pontificat de Pie XII, les durs effets de la censure romaine, et du caractère exceptionnel que revêt le moment conciliaire. Le matériau que choisit $\mathrm{D}$. Douyère est de toute première importance: le Père Congar, nommé expert au Concile, comme membre de la Commission théologique (il sera créé cardinal en 1994, à l'âge de 90 ans, par le pape Jean-Paul II), est un observateur savant et incisif qui bénéficie d'un vaste réseau d'informateurs, tant parmi les évêques que dans le milieu des théologiens. C'est aussi un acteur qui entend prendre parti afin de peser personnellement sur l'orientation des textes en débats et, in fine, sur les votes, en particulier dans les domaines de l'œcuménisme, de la collégialité épiscopale et de la place du laïcat. Ce que l'ouvrage de D. Douyère donne ainsi à voir, c'est le processus d'élaboration qui est à l'œuvre dans une vaste et complexe machine à penser et à dire le vrai qui fonctionne à la façon d'une série d'ateliers parallèles de réflexion et de production. Le résultat final, tel qu'il ressort des votes délibératifs, n'est pas pour autant ignoré, mais l'attention se porte délibérément sur le mouvement qui y conduit, et qui a d'ailleurs fait l'objet de plusieurs ruptures retentissantes, à commencer, on l'a vu, par celle qui a bouleversé le schéma De Ecclesia, revu en profondeur avant d'aboutir à la constitution dogmatique Lumen Gentium. L'auteur vise à comprendre, à travers le récit documenté et érudit du Père Congar, comment les pratiques communicationnelles de l'Église catholique «entrent en jeu dans l'élaboration dogmatique» (ibid.: 27), comment la communication et «l'expérience sociale de la parole» (ibid.: 72) font la doctrine de l'organisation. Le Père Congar rapporte ainsi les propos émis par Mgr Marty lors d'un colloque organisé en région lyonnaise: "Les évêques ont pris goût à se voir, à parler ensemble. On a beaucoup perdu, depuis des décades, à ne pas se voir » (ibid. : 73). L'intensité de tels échanges contraste avec la volonté du Vatican, celle en particulier du Saint-Office, de contrôler la communication, voire d'imposer le secret, intention dans laquelle le Père Congar décèle, pour la dénoncer, « un moyen d'atomiser et de neutraliser toute opposition» (ibid.: 83). 
La démarche de l'auteur se déploie en cinq étapes : considérer le journal en lui-même et préciser la lecture qui en est faite, selon une approche « expérientielle et cognitive " et non "lexicographique» (ibid.: 61); dessiner le contour des réseaux présents au Concile et considérer les échanges qui s'y déroulent; prêter attention au pouvoir de la parole, et en particulier à la qualité de l'expression orale, condition d'une écoute attentive; examiner le travail de l'écrit, c'est-à-dire la «façon de dire » pour "parler au monde » (ibid.: 114); enfin, analyser le lexique et le choix des mots, qui donnent souvent lieu à un combat serré commandé par la rigueur. Le soin accordé par le Père Congar au style, ordonné à une bonne accessibilité des textes, n'est pas dicté par la seule conception qu'il se ferait de la capacité de compréhension du lecteur chrétien lambda; il dit aussi s'être «aperçu souvent qu'une idée un peu forte, un peu fine, dépassait l'intelligence moyenne des évêques» (ibid.: 151). Cette observation a sans nul doute frappé $\mathrm{D}$. Douyère, qui la cite à deux reprises (également p. 160)... Pour illustrer l'importance que prend l'implication idéologique du choix des mots, l'auteur retient deux champs lexicaux, qui concernent respectivement les attributs de la Vierge Marie ( "co-rédemptrice» ?, "médiatrice » ?, «mère de l'Église» ?) et le qualificatif « déicide », longtemps affecté au peuple juif et qui sera finalement biffé, à l'encontre d'ailleurs du souhait initial du Père Congar.

L'analyse du «terrain de papier» est soumise à une approche méthodologique soigneusement argumentée, au point d'être "encadrée » par quatre textes: un préambule "Religion et communication " de près de vingt pages, une introduction, une conclusion et une longue postface, qui énonce "vingt indications pour l'étude du communicationnel pensé et pratiqué par le religieux» (ibid.: 223). Dans cet ultime texte, on lit notamment, au rang des prescriptions à visée déontologique, la nécessité pour le chercheur de faire preuve d'une extrême clarté sur ses propres positions confessionnelles afin d'éviter toute collusion avec son travail d'analyse; il importe dès lors que "cette inscription religieuse ou spirituelle éventuelle du chercheur soit spécifiée » (ibid.: 226). L'auteur renvoie à ce propos à l'un de ses propres articles où il dit avoir procédé à une telle explicitation, mais il n'en dit pas davantage. On peut le regretter dans la mesure où la recherche dont il livre les résultats procède d'une démarche que l'on pourrait qualifier de "compréhensive ». Or, le développement consacré dans le préambule à la «communication chrétienne » laisse planer un doute quant à l'éventuelle adhésion de l'auteur à ce qu'il exprime de la conception de «dieu » en "régime chrétien ", notamment lorsqu'il écrit que «le christianisme vient [...] réparer un défaut de communication dans l'Homme. [...] Le dieu chrétien lui-même est communication entre ses trois personnes et trouve sa genèse et sa puissance dans cet échange" (ibid.: 21-22). En tout état de cause, l'usage raisonné de l'ouvrage du Père Congar - qui ne fait jamais lui-même usage du terme «communication»... - permet une pratique anthropologique ainsi médiatisée et offre un regard original sur un Concile Vatican II en train de se faire... et dont les fruits ne tarderont pas à susciter des appréciations controversées. 
Les actes du colloque international qui s'est tenu à Lyon en mai 2016 expriment la nature et donnent la mesure de telles controverses qu'on ne saurait imaginer plus précoces (Sorrel, 2017). Leur intérêt tient notamment à une double énigme: pourquoi, cinquante plus tôt, le 24 juillet 1966, au sortir précisément du Concile de Vatican II (1962-1965), le cardinal Ottaviani, pro-préfet de la Congrégation pour la doctrine de la foi, a-t-il secrètement adressé aux présidents des conférences épiscopales une lettre s'alarmant des «abus grandissants dans l'interprétation de la doctrine du Concile » ? Et, dès lors, comment interpréter les réponses des évêques, dont la position et la fonction viennent de faire l'objet d'une profonde transformation du fait d'une nouvelle définition des relations entre primauté pontificale et collégialité épiscopale ? L'ouvrage se présente ainsi comme une investigation universitaire à caractère historique sur un examen romain à visée doctrinale. Il fait en cela œuvre novatrice: en effet, la lettre de juillet 1966 n'avait guère, jusqu'alors, retenu l'attention des chercheurs. Ce relatif oubli a pu tenir à la personnalité même du signataire de la lettre, connu pour être on ne peut moins favorable à l'aggiornamento conciliaire et donc estimé par avance rétif à l'égard de ses premiers effets. Ce courrier pouvait ainsi apparaître comme la traduction d'une posture de revanche ou de combat d'arrière-garde, perdu d'avance et donc sans réel enjeu. Les initiateurs de la réflexion collective dont l'ouvrage rend compte font au contraire le pari, assurément gagné, du caractère hautement significatif de ce document, qui constitue à leurs yeux, pourrait-on dire, un analyseur institutionnel des plus pertinents. Pour donner corps à leur exploration, ils font d'ailleurs le choix d'émailler les contributions de nombreuses annexes, pas moins de quatorze au total: ces sources primaires, qui représentent le plus souvent des réponses collectives ou individuelles à la lettre romaine, prennent la forme d'un véritable dossier documentaire.

En introduction, Christian Sorrel et Alessandro Santagata observent que la lettre du cardinal Ottaviani « résonne comme un syllabus errorum extensif, mais sans cible identifiée » (ibid.: 10-11), c'est-à-dire sans adversaire nommément désigné. Ce document énonce en effet dix opinions ou jugements estimés affecter le dogme et les fondements de la foi, et qui constituent le thème d'autant de questions posées aux épiscopats nationaux. Une telle "résonnance » se lit explicitement dans les réponses des épiscopats belge et hollandais, qui regrettent cette tonalité suspicieuse. Les "erreurs » et les "dangers» que souligne le document romain ont trait tout à la fois au statut de la révélation, à l'autorité du Magistère, à la théologie des sacrements, au péché originel, à la théologie morale... Cette critique explicite pose à l'évidence la question de l'identité de son rédacteur et/ou de son (ou de ses) inspirateur(s). En l'occurrence, quel rôle a joué le pape Paul VI, dont sont connues les indéniables marques d'estime à l'égard du cardinal Ottaviani, qui fut son supérieur à la Secrétairerie d'État puis à la Congrégation du Saint-Office, mais à qui il demandera néanmoins, en janvier 1968, de donner sa démission. P. Chenaux, qui rappelle ces relations passées, fait sienne l'hypothèse d'une convergence de vues entre Paul VI et son ancien mentor "sur la nécessaire défense de l'intégrité de la foi face aux remises en cause du Post-Concile» (ibid.: 81). 
Avant de rendre compte des positions des épiscopats nationaux, l'ouvrage s'attache à présenter les «contextes » et les «conjonctures » dans lesquels s'est inscrite la missive. Sur ce terrain, Étienne Fouilloux invite à ne pas tenir la lettre du cardinal Ottaviani pour une simple «survivance de la période préconciliaire» (ibid.: 65), mais à y voir bien plutôt la traduction d'un malaise du fait de la montée, à Rome, d'un climat d'inquiétude qui se nourrit de la perception de l'évolution culturelle du monde catholique. L'attention portée par Marialuisa Lucia Sergio aux archives du Conseil pontifical pour les laïcs lui permet ainsi de prendre la mesure des résistances suscitées par les projets de reconnaissance du rôle du laïcat et par le réaménagement de ses rapports avec une hiérarchie dont la lettre du cardinal Ottaviani non seulement réaffirme, mais spécifie avec force la fonction et la responsabilité : celles «de veiller, de diriger et de promouvoir le mouvement de rénovation commencé par le Concile». Sur ce plan, le cardinal ne manque probablement pas d'alliés: sans doute, en effet, l'épiscopat réformiste lui-même n'est-il "pas encore prêt à partager avec les laïcs le monopole de la réflexion doctrinale» (ibid.: 139).

L'un des points clés et indiscutablement novateurs de la lettre du cardinal Ottaviani réside dans la nature institutionnelle de ses destinataires: non pas l'ensemble des évêques considérés individuellement, comme il était de coutume avant le concile, mais bien, on l'a vu, les présidents des conférences épiscopales, instances que le "décret conciliaire sur la charge pastorale des évêques dans l'Église» (Christus Dominus) du 28 octobre de l'année précédente avait instituées et dotées d'une place toute nouvelle dans l'organigramme catholique. Certaines de ces instances, à l'instar de la Conférence des évêques de France, s'étaient déjà par avance réunies à Rome, en marge des sessions du Concile, mais la lettre romaine de juillet 1966 vaut pour l'ensemble d'entre elles reconnaissance officielle. La lettre représente du même coup une sorte de baptême du feu; il est en effet demandé aux nouvelles instances de se prononcer sur chacun des dix points névralgiques retenus par le cardinal Ottaviani. Pareille requête pouvait représenter un piège ou un écueil; les contours de l'exercice ainsi imposé sont en effet très clairement définis, et les réponses sinon dictées du moins pré-formatées... Il importe dès lors à l'observateur d'être attentif à la liberté d'expression et, le cas échéant, aux écarts que s'autorisent les épiscopats nationaux, ou, plutôt, la majorité qui s'exprime en leur sein. D. Pelletier est fondé à parler d' " une double mise à l'épreuve de la collégialité » (ibid.: 393): collégialité entre évêques dans le cadre d'un sorte de régime d'assemblée, et collégialité avec Rome, qui, pour ainsi dire, reste à la manœuvre en demeurant le pôle central de l'évaluation postconciliaire.

Les réponses qu'analysent les auteurs émanent de dix pays: France, PaysBas, Belgique, Allemagne, Suisse, Italie, Espagne, Hongrie, Canada et Argentine. L'étude que C. Sorrel consacre à la réponse de l'épiscopat français, «la seule qui ait fait l'objet d'une publication officielle» (ibid.: 143), quelques semaines seulement après son envoi, permet de suivre le processus, chaotique et incertain, de la consultation. Ce processus mobilise tout à la fois le Conseil permanent, le Bureau d'études doctrinales et pastorales et l'assemblée plénière; y sont 
également conviés, à l'initiative de Mgr Veuillot, coadjuteur du cardinal Feltin, archevêque de Paris, des théologiens ainsi que le Conseil du monde intellectuel du diocèse de Paris, créé deux ans plus tôt. Mgr Veuillot, qui exerce en l'affaire un véritable leadership, soumet un premier rapport à l'assemblée plénière de l'épiscopat français d'octobre 1966, la première depuis la clôture du Concile. La lettre du cardinal Ottaviani aura induit une forme d'apprentissage accéléré, dans un contexte de controverse, et représenté un test des relations avec Rome: au total, on voit à l'œuvre «un épiscopat peu disposé à entériner sans débat les suggestions du cardinal Ottaviani » (ibid.: 176). On peut d'ailleurs lire dans le refus d'organiser la réponse selon le plan en dix points fortement proposé par la lettre de juillet 1966 le désir d'affirmer un point de vue doctrinal original et singulier, qui se conjugue toutefois avec «la volonté de rassurer la Curie» (ibid.: 189). La liste des 38 experts sollicités au cours de la phase préparatoire témoigne du soin apporté à la préparation de la réponse de l'épiscopat français, dont les trois quarts des membres sont intervenus à l'une des phases de la consultation.

Les réponses des autres épiscopats nationaux qui font l'objet d'une analyse sont marquées par la diversité des postures et des méthodes. La réponse belge fut préparée, sur la suggestion du cardinal Suenens, par la Commission doctrinale de la conférence épiscopale à laquelle se sont joints les experts au Concile. Elle fut en large partie rédigée par Mgr Philips, qui venait de puissamment contribuer à l'élaboration des diverses versions de la constitution dogmatique Lumen Gentium. La distance critique qu'inspire à l'épiscopat belge la tonalité du document romain se perçoit clairement: à l'encontre d'une "attitude purement négative », la réponse assure qu' " une façon positive d'agir est nécessaire» (ibid.: 251). En Allemagne, c'est également la commission pour les questions de doctrine et de morale de la conférence épiscopale qui a joué un rôle central dans la réponse; Franz Xavier Bishof, pour qui le questionnaire "pouvait être interprété comme une motion de censure à l'égard des évêques des différents pays» (ibid.: 254), retient de la lettre adressée par le cardinal Döpfner au cardinal Ottaviani le vœu selon lequel le Saint-Siège devrait désormais renoncer à dresser "un nouvel inventaire des doctrines fausses et erronées» (ibid.: 262). En Suisse, du fait sans doute d'une absence de consensus, les évêques ont été invités à adresser des réponses individuelles. Une véritable synthèse s'est également révélée impossible en Argentine et en Espagne, où la dictature constituait alors un facteur majeur de clivage du milieu catholique. En ce qui concerne la réaction de l'épiscopat hongrois, András Fejérdy souligne une double difficulté: le contrôle exercé par le régime communiste sur l'Église et, d'ailleurs de ce fait, l'absence d'une théologie spécifiquement hongroise. En Italie, où le président de la conférence épiscopale a été nommé par Paul VI, Alessandro Santagata souligne "l'émergence d'une contestation sur la liberté d'expression théologique» (ibid.: 305), qui conduit certaines composantes du milieu catholique à voir dans les positions adoptées par Paul VI l'amorce d'une «restauration ». Le retard de l'envoi de la réponse canadienne, adressée neuf mois après la date requise, porte sans doute la marque d'un certain 
désintérêt; selon Gilles Routhier, la Congrégation pour la doctrine de la foi, qui «avait essuyé plusieurs critiques au moment du Concile, [...] ne semblait plus impressionner comme avant» (ibid.: 359).

Parmi les réponses analysées, on aura noté la surreprésentation de l'Europe et l'absence des continents africain et asiatique. L'échantillon qu'offre l'ouvrage permet toutefois de tirer au moins deux enseignements caractéristiques du lendemain du Concile: d'une part, l'inégale aptitude des épiscopats à se doter de méthodes collectives de travail et de réflexion pastorales et doctrinales; en outre, la rémanence d'une vive méfiance romaine vis-à-vis des effets réels ou supposés de l'aggiornamento. Cette méfiance, qui se fait l'écho d'inquiétudes prégnantes, se traduira dans la lettre encyclique de Paul VI Humanae Vitae sur le mariage et la régulation des naissances qui, publiée exactement deux ans après la lettre du cardinal Ottaviani, suscitera de très vives et durables oppositions.

Les ouvrages qui sont ici présentés ne couvrent évidemment pas l'ensemble des domaines où sont appréhendables les formes que prend le gouvernement de l'Église catholique: ni dans le temps, ni dans l'espace, et pas davantage au plan des nombreuses instances que compte l'institution. Il s'en dégage cependant deux enjeux essentiels, d'ailleurs en forte interaction l'un avec l'autre, liés respectivement à la dimension hiérarchique de l'institution et à la fonction magistérielle de la doctrine. Sur la base des données qu'ils livrent et des approches méthodologiques qu'ils mettent en œuvre, et parce que l'histoire continue..., ils invitent à prolonger les investigations afin, notamment, de mieux inscrire les controverses et les transformations actuelles dans le temps long. Ces investigations ne sauraient être conduites sans qu'une attention soit également portée aux changements socio-politiques et culturels dans lesquels se développent, ad intra comme ad extra, les débats relatifs au fonctionnement du champ catholique, entre orthodoxie et orthopraxie. À titre d'application pratique, et pour rester sur le terrain de l'analyse de l'action et des prises de position de l'épiscopat français, retenons une seule question. Peut-on légitimement opérer un lien entre, d'une part, le discours très remarqué qu'a prononcé le Président Macron à l'invitation de la Conférence des évêques de France au Collège des Bernardins le 9 avril 2018, discours qui sollicitait explicitement l'expression par l'Église de ses choix éthiques en matière de procréation $^{1}$, et, d'autre part, la forme de la publication (Évêques de France,

1. Le Président Macron a notamment déclaré aux représentants de la Conférence des évêques de France: «J'entends l'Église lorsqu'elle se montre rigoureuse sur les fondations humaines de toute évolution technique ; j'entends votre voix lorsqu'elle nous invite à ne rien réduire à cet agir technique dont vous avez parfaitement montré les limites.» Voir: http://discours.vie-publique.fr/ notices $/ 187000747 . \mathrm{html}$ 
2018: 112), le 20 septembre suivant, par cette même Conférence, d'un long texte signé, fait rare, par tous les évêques de France et qui, peut-être avec une assurance et une légitimité retrouvées, présente les obstacles éthiques à la modification de la loi en matière de procréation médicalement assistée?

\author{
Jacques PALARD \\ Centre Émile-Durkheim (Sciences Po Bordeaux) \\ j.palard@sciencespobordeaux.fr
}

\title{
Bibliographie
}

Chopelin Paul (éd.), 2017, Gouverner une Église en révolution. Histoires et mémoires de l'épiscopat constitutionnel, Lyon, Laboratoire de Recherche Historique Rhône-Alpes, coll. «Chrétiens et Sociétés. Documents et Mémoires».

Congar Yves, 2002, Mon journal du Concile, t. 1, Paris, Éditions du Cerf.

Douyère David, 2018, Communiquer la doctrine catholique. Textes et conversations durant le concile Vatican II d'après le journal d'Yves Congar, Genève, Labor et Fides, coll. «Enquêtes».

Évêques de France, 2018, La dignité de la procréation. PMA - Révision de la loi bioéthique, Paris, Bayard/Éditions du Cerf/Mame.

Jankowiak François, Pettinaroli Laura (éds.), 2017, Les cardinaux entre Cour et Curie. Une élite romaine, 1775-2015, Rome, École française de Rome.

LAGroye Jacques, 1983, «L'épiscopat et la société française », in Bravasa É., Emeri C., Seurin J.-L. (éds.), Religion, société et politique: mélanges en hommage à Jacques Ellul, Paris, Presses universitaires de France.

MacMullen Ramsay, 2008 [2006], Voter pour définir Dieu. Trois siècles de Conciles (253-553) [Voting about God in early church councils], Paris, Les Belles Lettres.

Plongeron Bernard, 1973, Théologie et politique au siècle des Lumières (1770-1820), Genève, Droz, 1973

-, 1989, L'Abbé Grégoire (1750-1831) ou l'Arche de la fraternité, Paris, Éditions Letouzey et Ané.

SORREL Christian (éd.), 2017, Renouveau conciliaire et crise doctrinale. Rome et les Églises nationales (1966-1968), Lyon, Laboratoire de Recherche Historique Rhône-Alpes, coll. "Chrétiens et Sociétés. Documents et Mémoires ".

VAldrini Patrick, 1988, «Exercice du pouvoir et principe de soumission », Concilium, 217, p. 119-127.

VARIN Roland, 2016, Le sacrement du ministère apostolique. La sacramentalité de l'épiscopat et ses conséquences ecclésiologiques, Paris, Artège Lethielleux. 